\title{
Actualización de protocolos de cardiología nuclear para evaluación y manejo de enfermedad coronaria. Sociedad Chilena de Cardiología y Cirugía Cardiovascular.
}

\author{
Teresa Massardo, Rodrigo Jaimovich, José Canessa, Gabriel Castro*, Juan Ramón Soto. \\ Departamento de Imágenes Cardiovasculares No Invasivas. \\ Sociedad Chilena de Cardiología y Cirugía Cardiovascular. \\ ${ }^{* B e c a r i o}$ Programa de Medicina Nuclear, Hospital Clínico Universidad de Chile
}

\section{Resumen:}

Se revisan los protocolos de cardiología nuclear actuales, con énfasis en los estudios tomográficos de fotón único ("SPECT") de perfusión miocárdica principalmente en evaluación de enfermedad corona- ria (EC). Las indicaciones y protocolos más utilizados son detallados, así como la actualización bibliográfica pertinente, tanto al uso clínico como al control de calidad e interpretación de los estudios.

Palabras clave: SPECT, perfusión miocárdica, enfermedad coronaria.

\section{Introducción:}

Se revisan los protocolos de cardiología nuclear, que han sido ya publicados en esta revista en los años 1996 y 2004, con énfasis en los estudios tomográficos de fotón único SPECT de perfusión miocárdica en evaluación de enfermedad coronaria (EC) ${ }^{1}$. Es necesario conocer las fortalezas y debilidades de las técnicas isotópicas no invasivas debido a la reciente aparición de tecnologías de imágenes con y sin uso de radiaciones ionizantes como la tomografía computada con score de calcio o la resonancia magnética funcional que así como la ecocardiografía de estrés entregan diversos parámetros complementarios en la aproximación a la EC.

En el país existen múltiples equipos SPECT multicabezal con software comerciales que entregan parámetros cuantitativos de la perfusión miocárdica gatillada electrocardiográficamente que permiten análisis de extensión e intensidad de los defectos así como de valores de función ventricular izquierda.

En este campo es importante asegurar lo siguiente ${ }^{2-5}$ : a) una adecuada indicación de los estudios isotópicos, con selección del protocolo específico.

b) optimizar adquisición, procesamiento e interpretación para lograr un informe reproducible y que cumpla los requerimientos del tratante.

c) efectuar procedimientos con mínimo riesgo y bajas dosis de irradiación a los pacientes y personal involucrado. En estudios con fines diagnósticos, pronósticos y de evaluación de terapia se requiere reconocer la presencia de isquemia, utilizando protocolos que logren un consumo miocárdico de oxígeno adecuado y alcanzando frecuencias cardíacas mayores a $85 \%$ de la máxima teórica; en los casos en que sea difícil lograr esto, se requiere usar vasodilatadores i.v. y frente a su contraindicación, inótropos positivos. Lo ideal es preferir el ejercicio graduado en cinta ergométrica como agente de estrés, a lo que se asocia el valor agregado intrínseco del estudio de ergometría. En caso de usar vasodilatadores pudiera ser aconsejable, en algunos casos, agregar ejercicio de baja carga para reducir los efectos colaterales indeseados y aumentar el consumo de oxígeno ${ }^{6}$.

Correspondencia: Teresa Massardo M.D.

Sección Medicina Nuclear,

Hospital Clínico Universidad de Chile

Santos Dumont 999-1E, Independencia,

Santiago, Chile.

Zip Code: 6531063

Fono Fax (562) 7770569

E-mail: tmassardo@redclinicauchile.cl 
Indicaciones de los estudios de perfusión miocárdica spect

A.- Diagnóstico de EC: La principal indicación es el tamizaje en poblaciones de riesgo clínico intermedio de EC (hombres de edad mediana con factores de riesgo tradicionales presentes y prueba de esfuerzo no concluyentes o con dolor precordial atípico y mujeres perimenopáusicas con prueba de esfuerzo y/o dolor precordial no concluyente), con el fin de seleccionar en forma más adecuada los pacientes que serán derivados a estudio invasivo del árbol coronario. La sensibilidad y especificidad de los estudios SPECT de perfusión con estrés en diagnóstico de EC es de 80-85\%, en diversos trabajos con amplia casuística. Existen ciertos sub-grupos de pacientes, como los con enfermedad ateroesclerótica vascular periférica que debieran considerarse como poblaciones especiales por su mayor riesgo de EC y que corresponden a los diabéticos asintomáticos, insuficientes renales crónicos, ancianos y aquellos con factores de riesgo no tradicionales añadidos. ${ }^{2,4,7-9}$

Los artefactos de atenuación por tejidos blandos en las imágenes miocárdicas pueden producir falsos positivos que, en parte, se disminuyen utilizando gatillado electrocardiográfico, lo que permite reconocer territorios normales que presenten adecuado engrosamiento sistólico a pesar de mostrar menor actividad o captación de los trazadores radiactivos en las imágenes no gatilladas. Esto se observa con cierta frecuencia en pared anterior en mujeres y en pared diafragmática en hombres. En el aspecto técnico se debe considerar la opción del uso hardware y software de corrección de atenuación, que intenta eliminar estos artefactos; sin embargo, no siempre está disponible, es de costo elevado, tiene el riesgo de sobre-corregir y por lo tanto de generar falsos negativos, lo que clínicamente puede ser más significativo como error de interpretación del resultado final. Algunos grupos efectúan la adquisición del estudio SPECT con el paciente en decúbito prono además del decúbito dorsal tradicional, para evaluar principalmente pared inferior, aunque esto alarga el tiempo de examen lo que puede solucionarse con equipos de 3 cabezales, no disponibles en nuestro medio.

B.- Evaluación pronóstica: En este importante aspecto los estudios de SPECT miocárdico tienen gran utilidad en la práctica clínica. Se acepta que una perfusión en estrés normal puede predecir con certeza, una baja incidencia de eventos cardíacos mayores a largo plazo, bajo un $1 \%$ al año, especialmente si se usa esfuerzo como estresor. La validez de esta afirmación es, al menos, por 2 años, puesto que la EC es progresiva, aunque en diabéticos o insufi- cientes renales este plazo es más corto y la incidencia es mayor, del orden del 3\%-5\%, por la mayor severidad y extensión de la ateroesclerosis coronaria. Si el estudio de perfusión es anormal, la tasa de eventos cardíacos anual es sobre $6 \%$, sobre todo si se demuestra isquemia significativa ${ }^{8,10}$.

C.- Evaluación de la repercusión funcional de EC conocida y estratificación de riesgo: Esta es otra gran indicación, tanto para determinar extensión y severidad de la EC, como para definir conducta terapéutica médica o de revascularización coronaria. Si se efectúa adquisición gatillada de estas imágenes se agrega valor pronóstico al evaluar simultáneamente parámetros de función sistólica ventricular izquierda, ampliamente validados. El estudio de SPECT miocárdico anormal tiene valor incremental para identificación de muerte de origen cardíaco. Un defecto de perfusión de grado leve post ejercicio predice un bajo riesgo de muerte de origen coronario, con riesgo intermedio para infarto de miocardio no fatal. El paciente podría beneficiarse con un manejo clínico no invasivo ${ }^{(11)}$.

D.- Detección de EC y evaluación de riesgo coronario en pacientes que serán sometidos a cirugía no cardíaca: Pacientes con riesgo de EC como son los pacientes con enfermedad arterio-esclerótica periférica que deban ser sometidos a intervenciones de mediano o alto riesgo anestésico y/o quirúrgico, pueden ser evaluados con seguridad mediante SPECT de perfusión miocárdica preoperatoria con protocolos de provocación de isquemia, que en general es de tipo farmacológica.

E.-Infarto de miocardio (IM): En este caso, los estudios de perfusión miocárdica sirven tanto para confirmar presencia y magnitud de la lesión cicatrizal miocárdica, reconocer y cuantificar isquemia residual y los territorios coronarios involucrados, detectar y cuantificar la presencia de viabilidad miocárdica, evaluar resultados de revascularización quirúrgica o percutánea y también establecer pronóstico en casos de IM antiguo.

Es conocido que post-infarto, coexisten en el miocardio dependiente del vaso coronario afectado: zonas de necrosis, isquemia crónica, hibernación, atontamiento (especialmente, si hubo reperfusión precoz) e incluso áreas con perfusión normal. La revascularización permite mejorar la función regional y global del ventrículo izquierdo y puede mejorar la sobrevida a largo plazo. Es por esto que los métodos de diagnóstico no invasivos con capacidad de pesquisar viabilidad miocárdica cobran importancia.

El uso de tomografía de positrones PET con un trazador análogo de glucosa marcado con flúor18, la desoxiglucosa (FDG) tiene relativo mayor rendimiento que el SPECT de perfusión con Sestamibi-Tecnecio ${ }^{99 m}$ ó Talio201, y 
otras técnicas no invasivas como la ecografía de estrés con dobutamina con protocolo de doble fase o la resonacia magnética. La glucosa es la principal fuente de energía del miocárdico en condiciones de hipoxia celular, así como los ácidos grasos de cadena corta lo son en condiciones normales. La acumulación de FDG en miocardio hipoperfundido y con alteración de motilidad permite asegurar la presencia de tejido viable y otorga al paciente la posibilidad de la revascularización con buen resultado funcional. Esta técnica se encuentra disponible en el país aunque una de sus limitantes es su alto costo relativo. En cuanto al pronóstico post IM, la presencia de isquemia residual significativa es un factor de riesgo conocido, así como la isquemia coexistente en territorios coronarios alejados. El tamaño del área infartada y la presencia de viabilidad significativa en ésta son también factores pronósticos independientes.

F.- Síndrome coronario agudo: En algunos centros de alta complejidad y con disponibilidad de recursos se utiliza el SPECT de perfusión miocárdica para ayudar a decidir el ingreso a unidades coronarias, en pacientes con dolor precordial agudo, que tengan probabilidad clínica baja o intermedia de EC y en quienes se desee descartar un infarto de miocardio en curso o acidente de placa y con diagnóstico sea indeterminado mediante otros exámenes (enzimas cardíacas y ECG). El seleccionar adecuadamente estos pacientes es un problema serio y relativamente frecuente ${ }^{12}$. En un trabajo efectuado en una unidad de emergencia, de comparación de 2 grupos con más de 1000 pacientes cada uno con sospecha de isquemia miocárdica aguda, sin anormalidades obvias en el ECG efectuando enfoque habitual y Sestamibi en reposo, se concluyó que las imágenes isotópicas reducen hospitalizaciones innecesarias, sin disminuir la tasa de ingreso en quienes sí la requieren por isquemia aguda ${ }^{13}$. También se puede usar prueba de estrés de perfusión miocárdica SPECT con buen valor pronóstico y baja tasa de eventos a largo plazo en los pacientes con test de negativo para isquemia ${ }^{(14)}$. En estos pacientes se pueden utilizar imágenes en reposo con Sestamibi y obtener información de existencia, localización, extensión y severidad de un eventual infarto o lesión isquémica con atontamiento. Sin embargo, se requieren equipos e insumos en las unidades de emergencia y personal permanentemente disponible. El Sestamibi, además, por su mínima redistribución en el miocardio, permite estudiar al paciente inyectando el trazador en fase aguda y aplazando las imágenes en algunas horas, hasta después de obtener su estabilización.

G.- Miocardiopatía isquémica: Es una patología más frecuente de lo sospechado entre portadores de insuficiencia cardíaca congestiva. La revascularización coronaria completa en sujetos que aún tengan músculo viable es una alternativa terapéutica al trasplante cardíaco. Los protocolos de perfusión miocárdica SPECT orientados a detectar con la máxima sensibilidad la presencia de viabilidad residual permiten reconocer territorios viables e isquémicos. Sin embargo, el diagnóstico diferencial con miocardiopatía no isquémica puede ser difícil y se deben buscar indicadores indirectos de isquemia balanceada o subendocárdica. Entre estos están: el índice de dilatación transitoria en estrés en ventrículo izquierdo dilatado. Está claro que en pacientes con fracción de eyección ventricular izquierda (FEVI) muy reducida (menor al 25\%), sólo se pueden utilizar con seguridad protocolos de perfusión en reposo, sin estrés asociado $^{(15)}$.

\section{Técnica:}

\section{Equipos:}

1. Gamacámara SPECT: Se basa en un cristal de ioduro de sodio que emite luz al ser incidido por radiación gamma procedente del paciente, a quien se le ha administrado un radiofármaco, la señal luminosa se convierte en impulso eléctrico y posteriormente en señales digitales. Los estudios de cardiología nuclear se realizan principalmente con equipos tomográficos SPECT, los que al igual que los de tipo planar, utilizan la señal electrocardiográfica y dividen cada ciclo cardíaco (onda $\mathrm{R}$ ) en varios fragmentos temporales lo que permite el análisis de función ventricular izquierda. Los equipos con más de un detector tienen ventajas pues permiten estudios más cortos con menor probabilidad de movimiento del paciente durante la adquisición y además menor dosis. El SPECT asociado a un equipo de tomografía computada o SPECT-CT, llamado también equipo híbrido logra corregir atenuación por tejidos blandos lo que produce artefactos que disminuyen la especificidad del examen en presencia de EC.

Análisis. Para evaluar la perfusión miocárdica se usan diversos parámetros, tales como:

-Análisis visual de cine-loop de la adquisición original que permite relacionar tamaño de cavidades ventriculares y dilatación en las fases analizadas y reconocer movimiento durante la adquisición con eventual corrección posterior usando software adecuado.

- Índices semi-cuantitativos de captación que consideran actividad en pared normal del ventrículo izquierdo (VI) respecto a la existente en el campo pulmonar, usando áreas de similar tamaño, para pesquisar sobrecarga ventricular, en forma simple en fase post-estrés, más útil con ejercicio y con Talio que con vasodilatadores y MIBI. 
Otro índice es la dilatación transitoria del VI en estrés respecto al reposo (Transient Ischemic Dilation o TID), que indica isquemia subendocárdica

-Índices obtenidos de programas de software comercial disponibles para SPECT, que usan algoritmos matemáticos para cuantificar los hallazgos y comparar con bases de datos de sujetos normales o con baja probabilidad de EC 16, 17. Asimismo, existen parámetros de perfusión y de función en los estudios gatillados, entregados en forma automática o con mínima intervención del operador. Se puede evaluar la extensión de los defectos reversibles [isquemia] y fijos [infarto o isquemia severa, o hibernación] o mixtos; la motilidad y engrosamiento sistólico, las curvas de volumen, fracción de eyección de ventrículo izquierdo (FEVI) y volúmenes ventriculares e incluso la excentricidad de la cavidad (indicador de remodelación ventricular). Se utiliza también la relación entre volumen de fin de diástole (VFD) en el post estrés y en reposo para pesquisar disfunción ventricular izquierda transitoria. El ventrículo izquierdo se analiza en base a segmentos de un mapa polar estandarizado con 17 o 20 de ellos ${ }^{18}$. Existe la opción de efectuar la imagen post-estrés inmediatamente terminado el ejercicio y posterior a la estabilización del paciente lo que permite contar con parámetro pronóstico adicional por caída de la FEVI asociado a dilatación transitoria incluso independiente de la presencia de defectos isquémicos ${ }^{(19)}$. Sin embargo, con dipiridamol puede haber exceso de actividad esplácnica que dificulta esta evaluación. En general se prefiere comenzar la adquisición a los 30 min posterior a efectuada la inyección en estrés. Aún puede existir atontamiento isquémico residual en ese lapso y debe ser considerado incluyendo la búsqueda de alteraciones de motilidad ventricular izquierda segmentarias o difusas.

Los programas Cedars Quantitative Perfusion $\left(\mathrm{QPS}^{\circledR}\right)$, Quantitative Gated $\left(\mathrm{QGS}^{\circledR}\right)$, Quantitative Blood Pool $\left(\mathrm{QBS}^{\circledR}\right)$ y Emory Toolbox ${ }^{\circledR}$ son los más difundidos. Los índices o scores más comunes para perfusión corresponden a summed stress score, summed rest score y summed difference score (SSS, SRS y SDS, respectivamente), así como la extensión porcentual de los defectos fijos o reversibles. Los parámetros funcionales más usados son la FEVI y los volúmenes telediastólico y telesistólico (VFD y VFS) y el índice de dilatación transitoria de VI. Además, hay nuevos indicadores de isquemia que consideran tanto la extensión como la severidad de los defectos de perfusión (TPD) y también de forma y excentricidad ventricular izquierda ${ }^{20,21}$.

2. Equipos PET (Positron Emission Tomography): Son necesarios para estudios con emisores de positrones, como Flúor, Oxígeno, Carbono y Nitrógeno marcados, muy útiles para evaluar diversos aspectos del metabolismo miocárdico y del flujo sanguíneo coronario. Estos equipos pueden ser específicos para cada elemento o bien pueden ser "híbridos", y ser utilizados para distintos elementos; actualmente están asociados a un equipo CT, pero en el futuro próximo a una resonador magnético (RM), con el fin de localizar mejor los territorios por la mayor resolución anatómica que entregan el CT y RM, y efectuar corrección de atenuación. También se pueden gatillar y analizar cuantitativamente. Puede ser necesario comparar estas imágenes de PET con las de estudios de perfusión SPECT para una correcta interpretación si no se tiene disponible un estudio de flujo coronario con nitrógeno o rubidio.

\section{Radioisótopos en SPECT Miocárdico}

Talio ${ }^{201}$ : El cloruro de Talio ( $\mathrm{Tl}^{201}$, vida media $72 \mathrm{~h}$ ) es un análogo del potasio, ingresa por trasporte activo a la célula miocárdica mediante la bomba de $\mathrm{Na}-\mathrm{K}$, con tiempo de residencia intracelular de aproximadamente 4-5 h. Se administra i.v., con dosis entre 111-185 MBq (3-5 mCi). La extracción miocárdica en primer paso es mayor a $85 \%$; presenta una cinética de reingreso o "redistribución". Se considera un marcador bastante fidedigno de flujo sanguíneo coronario regional y de indemnidad de membrana celular. Su captación es relativamente proporcional a la viabilidad miocárdica (actividad bajo 30\% del máximo correspondería a tejido con baja probabilidad de recuperación). $\mathrm{El} \mathrm{Tl}^{201}$ se puede reinyectar luego de imágenes de estrés para reconocer tejido viable que no captó en etapa inicial. El SPECT se puede gatillar especialmente si se cuenta con equipos multicabezal, pues la actividad es subóptima, por las características físicas del isótopo y por limitaciones de dosimetría del paciente. No está disponible permanentemente en la actualidad en el país.

Isonitrilos Tecneciados: El Sestamibi (MIBI) marcado con Tecnecio ${ }^{99 m}\left(\mathrm{Tc}^{99 \mathrm{~m}}\right.$, vida media $6 \mathrm{~h}$ ) tiene una energía más adecuada que el $\mathrm{Tl}^{201}$ para su detección con gamacámara SPECT. La eficiencia de extracción miocárdica de los derivados de isonitrilos es inferior a la de $\mathrm{Tl}^{201}$. Su acumulación intramiocito es también dependiente de flujo y de indemnidad de membrana, aunque tiene un importante componente de difusión pasiva; no tiene redistribución significativa, por lo que requiere doble inyección (estrés y reposo). Sus imágenes habitualmente se gatillan. También el grado de captación celular en reposo está relacionado con la viabilidad. Existen otros tecneciados, relativamente similares no disponibles en el país como la tetrofosmina. Las ventajas de las moléculas marcadas con $\mathrm{Tc}^{99 \mathrm{~m}}$ respec- 
to al $\mathrm{Tl}^{201}$ es que producen menor irradiación al paciente, tienen mejor calidad de imagen (indicación en obesos) aunque requieren mayor dosis. Asimismo permiten protocolos de imagen más versátiles como postergar la adquisición en casos de dificultades con el equipo o su repetición por movimiento del paciente durante la adquisición o interferencia de asas intestinales o vesícula, pues el Sestamibi se elimina por vía biliar, así como efectuar en cualquier secuencia la adquisición de las fases de estrés y reposo, tanto el mismo día como en días sucesivos.

Flúor18-desoxiglucosa (FDG): ( $\mathrm{F}^{18}$, vida media 110 min). Es una molécula análoga de la glucosa marcada, que ingresa a la mitocondria por trasportadores específicos de membrana; se detiene en la primera fase enzimática de la glicólisis y no alcanza al ciclo de Krebs. $\mathrm{El} \mathrm{F}^{18}$ es producido en ciclotrón y emite dos fotones gamma de $511 \mathrm{KeV}$, al aniquilarse por colisión con un electrón orbital, estos fotones simultáneos son detectados por la cámara PET. La técnica permite pesquisar viabilidad en territorios que presenten defectos de perfusión en imágenes SPECT o PET con trazadores de flujo. Los estudios PET FDG pueden gatillarse con ECG para derivar parámetros funcionales.

Existen otros radiofármacos PET para evaluar flujo coronario, no disponibles actualmente en nuestro medio como el agua marcada con $\mathrm{O}^{15}$ y el amonio con $\mathrm{N}^{13}$ cuya principal desventaja es su corta vida media. El $\mathrm{Rb}^{82}$ es muy preconizado en la actualidad pues permite evaluar isquemia, sin problemas de artefactos por atenuación y con mejor resolución que el SPECT; sin embargo, requiere un generador de alto costo. Mediante el uso de vasodilatadores se puede cuantificar flujo absoluto y reserva coronaria.

\section{Tipos de estrés utilizados en cardiología nuclear}

I -Protocolos de Ejercicio: Se utilizan los protocolos estándar de Bruce, Bruce modificado o Naughton en banda continua idealmente, o en su ausencia, en bicicleta ergométrica, similar a la prueba de esfuerzo tradicional (cuyas contraindicaciones son ampliamente conocidas). Se debiera evitar el beta-bloqueo y uso de antianginosos que enmascaren la isquemia o impidan alcanzar una frecuencia cardíaca satisfactoria o un consumo de oxígeno superior a 5 METS, con suspensión previa de al menos 24- 48 hrs [si esto no es posible se debe efectuar protocolo vasodilatador]. Al alcanzar los parámetros hemodinámicos máximos previstos según edad, se inyecta el radioisótopo seleccionado y se mantiene el ejercicio, al menos por $30-$ $60 \mathrm{seg}$ adicionales. Esta prueba debe ser monitorizada por un especialista a cargo y en un centro con equipamiento y entrenamiento en resucitación para seguridad del paciente. El nivel de ejercicio alcanzado influye en el rendimien- to de la prueba; cuando éste es inferior a 5 equivalentes metabólicos o METS y/o el 85\% de la frecuencia cardíaca máxima (FCMT) predicha según edad, disminuye la sensibilidad para pesquisar EC, incluso hasta en 15-20\%, afectando además la evaluación del grado de isquemia. Se puede administrar una dosis de atropina para llegar a la frecuencia objetivo; si esto no ocurre, es recomendable detener la prueba de esfuerzo, esperar la recuperación del paciente e iniciar un protocolo de estrés farmacológico.

\section{II-Estrés Farmacológico [Ver anexo]:}

A. Drogas de Acción Vasodilatadora: La más usada en el país por motivos de costo es el dipiridamol, vía e.v. Tanto ésta como la adenosina aumentan los niveles de adenosina endógena que es un potente vasodilatador no selectivo (A1, A2a, A2b y A3) que actúa en arterias coronarias, bronquios y otros territorios vasculares. A nivel de territorio coronario se obtiene aumento de flujo de 4 a 5 veces sobre el nivel basal; vasos enfermos, que ya han usado su reserva vasodilatadora, aumentan proporcionalmente menos el flujo respecto a los sanos y, en raros casos incluso pueden disminuirlo por robo desde territorios vecinos. La vasodilatación de otros territorios produce efectos no deseados como: cefalea, mareo y bochornos. Los efectos cardiovasculares pueden ser serios, aunque son tan poco frecuentes como en el test de esfuerzo convencional, además de autolimitados, siendo por lo tanto un protocolo demostradamente seguro ${ }^{(22,23)}$. El antagonista del dipiridamol es la aminofilina i.v. en dosis de $125-250 \mathrm{mg}$, que revierte rápidamente la sintomatología asociada al uso del dipiridamol, y que puede observarse, en general, hasta una hora post-administración, pero los efectos también pueden ser más tardíos. Este antagonista debiera inyectarse al menos 3-5 min después del radiofármaco para permitir su redistribución, captación y acumulación intramiocárdica, de lo contrario se invalida el estudio. Los vasodilatadores no deben emplearse en pacientes con obstrucción bronquial severa dependiente de xantinas. Entre las indicaciones de los vasodilatadores están todas las causas que impiden un adecuado nivel de ejercicio y el bloqueo completo de rama izquierda del Haz de His, que puede producir falsos positivos en la pared septal con ejercicio. La dosis i.v. de dipiridamol es de $0,56 \mathrm{mg} / \mathrm{kg}$ en infusión de 4 min., la que podría ser aumentada con leve mayor proporción de efectos indeseados para mejorar teóricamente la sensibilidad del estudio. La ventaja de la adenosina es que es mejor tolerada por su corta vida media que permite, con la sola suspensión de la infusión, la desaparición de los efectos colaterales. Existen nuevos vasodilatadores con acción selectiva sobre receptores de adenosina A2a recientemen- 
te aprobados en Estados Unidos de América, entre ellos Binodenoson ${ }^{\circledR}$ y Regadenoson ${ }^{\circledR}$ que otorgan algunas ventajas comparativas, pero su costo aún es alto.

-B. Drogas de Acción Inótropa: Entre estas, la dobutamina es el fármaco utilizado en la actualidad si es que existe indicación de estrés farmacológico e impedimento para uso de vasodilatadores. Obtiene un real aumento de la contractilidad, del consumo de oxígeno y del flujo coronario miocárdicos. Sus contraindicaciones son conocidas; su aplicación requiere infusión continua con dosis escalonada, según protocolos usados en ecocardiografía de estrés farmacológico y sus efectos colaterales cardiovasculares pueden ser serios, especialmente arritmias.

Protocolos de perfusión más empleados en la actualidad: 1) Con provocación de isquemia

-Tecnecio ${ }^{99 m}$-Sestamibi reposo-estrés o estrés-reposo. Las imágenes pueden obtenerse el mismo día o en días sucesivos. En los protocolos de un día se recomienda iniciar con el estudio en reposo a dosis bajas (296-370 MBq; 8-10 $\mathrm{mCi})$ y terminar con el estudio de estrés a dosis altas (925$1110 \mathrm{MBq} ; 25-30 \mathrm{mCi})$. En casos en que logísticamente convenga comenzar con el estrés, la dosis inicial igualmente debe ser la más baja. En protocolo de 2 días, ambas dosis debieran ser no mayores a $555 \mathrm{MBq}$ cada una (15 $\mathrm{mCi})$ para disminuir irradiación innecesaria del paciente. El nivel de dosis ionizante entregada por procedimientos diagnósticos cardiológicos es un tema de actualidad ${ }^{24}$, aunque la del estudio SPECT es menor al de tomografía computada de coronarias.

El tiempo entre la inyección y la adquisición de imágenes oscila entre 30 y $60 \mathrm{~min}$ con intervalo mínimo entre ambas inyecciones de $3 \mathrm{hrs}$. cuando el protocolo de estrés es el inicial, o bien períodos menores (incluso inmediatamente) si se logra diferencia de 3,5 a 4 veces la dosis basal en reposo. Se ha considerado efectuar primero la fase de estrés $\mathrm{y}$, si es normal, se podría omitir la fase de reposo con el fin de acortar el estudio y disminuir irradiación, aunque esta aproximación sólo debiera ser utilizada en casos de diagnóstico de EC preferentemente ${ }^{25}$.

-Tecnecio ${ }^{99 \mathrm{~m}}$-Sestamibi en estrés y en reposo post nitratos. Se sigue el protocolo anterior pero con la administración adicional de nitratos vía sublingual (nitroglicerina, 0,5-1,0 mg) u oral (dinitrato de isosorbide, $10 \mathrm{mg}$ ) a los $5 \mathrm{y}$ 15 min respectivamente antes de la inyección de Sestamibi en reposo, en casos con evidencia de infarto o defecto en estrés. Los nitratos producen máxima vasodilatación coronaria en reposo, aumentando la sensibilidad del estudio para detección de viabilidad miocárdica ${ }^{26,27}$.
- T1201 estrés-reinyección. Es útil para detectar isquemia y viabilidad miocárdica. La dosis para el estrés es de 111$148 \mathrm{MBq}(3-4 \mathrm{mCi})$, mientras que para la reinyección se emplea $37 \mathrm{MBq}(1 \mathrm{mCi})$, administrados inmediatamente después de efectuadas las imágenes de estrés o 1 hora antes de las imágenes tardías. Tiene la ventaja, para algunos grupos de hacer más eficiente la adquisición, obtener conteos comparables y evaluar viabilidad en todos los pacientes considerando la posible existencia de IM no conocidos. Produce mayor irradiación que los estudios con moléculas marcadas con $\mathrm{Tc}^{99 \mathrm{~m}}$.

-Doble isótopo T1201 reposo y Tc ${ }^{99 \mathrm{~m}}$ Sestamibi en estrés al día siguiente es un protocolo interesante, sin embargo, entrega mayor dosis de irradiación y tiene mayor costo asociado.

\section{2) Sin provocación de isquemia (sólo viabilidad)}

No requieren monitorización cardiológica y obviamente no es útil para detectar isquemia inducible.

a) $\mathrm{Tc}^{99 \mathrm{~m}}$-Sestamibi en reposo con nitratos. Se adquieren imágenes en reposo, 30-60 min después de la inyección de $\mathrm{Tc}^{99 \mathrm{~m}}$-Sestamibi a las dosis ya descritas. Se repite el procedimiento el mismo día, o al día siguiente, previa administración de nitratos oral o sublingual, en dosis habituales, con el fin de intensificar captación de células viables. Se puede efectuar cuantificación relativa de la captación miocárdica.

b) T1201 reposo-redistribución. Fue el protocolo inicial para viabilidad. Las imágenes se comienzan a los 10 min post inyección (111-148 MBq; 3-4mCi), mientras que las de redistribución se efectúan a las 3-4 hrs. Se pueden adicionar imágenes más tardías a las 12-24 hrs., aunque, por lo general no mejoran significativamente la sensibilidad.

c) Flúor18-deoxiglucosa FDG. El paciente debe estar en normoglicemia al efectuar la inyección de FDG. Aunque no es imprescindible, se han descrito varios métodos para optimizar la utilización de glucosa por el miocito. La carga de hidratos de carbono con o sin utilización de ácido nicotínico como hipolipemiante (bloqueador de la utilización de ácidos grasos) tiene excelente rendimiento y es fácilmente aplicable. El protocolo sugerido en pacientes no diabéticos incluye: a) dieta baja en grasas por $24 \mathrm{hrs}$ y ayuno al menos 4 hrs preinyección de FDG; b) $250 \mathrm{mg}$ de Acipimox $\left(\right.$ Olbetam $\left.{ }^{\circledR}\right) 3$ hrs antes de la inyección de FDG y 350 $\mathrm{mg}$ de ácido acetilsalicílico (bloqueador de efectos desagradables del Acipimox) 90 min antes de ésta; y c) comida de 75-g de carbohidratos 90 min antes de la inyección de FDG. En diabéticos se usa el mismo 
protocolo, pero no se requiere ayuno. Se debe realizar hemoglucotest previo a la inyección de FDG, la glicemia debiera estar bajo $140 \mathrm{mg} / \mathrm{dl}$ para inyectar el radiofármaco.

Interpretación de los estudios de imágenes isotópicas

\section{A. SPECT de Perfusión Miocárdica}

\section{A.1. Generales}

Se deben considerar varios aspectos, especialmente:

a) antecedentes del paciente y cuál es la pregunta del médico clínico que el examen debe responder.

b) radiofármaco y protocolo utilizado (adecuado para indicación del paciente).

c) si el protocolo fue cumplido en forma estricta (ej. Si es esfuerzo, si alcanzó la FCMT y METS objetivos o si es dipiridamol si suspendió las xantinas, uso de drogas con potencial interferencia en el resultado, etc)

d) control de calidad de la adquisición (equipo adecuadamente calibrado, posición similar del paciente en ambas fases, movimientos durante la adquisición, gatillado ECG sin arritmia).

e) Si alguna de estas condiciones no fuera la requerida se debe reconsiderar la repetición de la o las fases con problemas o eliminar el análisis del gatillado.

f) control de calidad del análisis de los datos, especialmente reorientación y ejes del ventrículo izquierdo, filtros y suavizados adecuados y de acuerdo a la dosis y radiofármaco.

g) por otra parte, se debe observar posible dilatación o hipertrofia del ventrículo derecho y captación focal de masas tumorales mediastínicas o pulmonares como hallazgo adicional.

\section{A.2. Perfusión}

a) captación homogénea en las paredes del VI sin cambio entre las fases de estrés y reposo, se interpreta como normal.

b) defectos fijos regionales en ambas fases, son concordantes con infarto de miocardio, sin embargo, también podrían corresponder a atenuación o miocardio hibernado. De acuerdo a los antecedentes clínicos será necesario estudio para viabilidad.

c) defecto reversible, es decir, defecto en estrés que se normaliza o disminuye significativamente en reposo, es explicable por isquemia; también pueden ser debidos a atenuación con diferencia de posicionamiento del paciente en las adquisiciones.

d) defectos parcialmente reversibles: infarto de miocardio con isquemia residual.

e) defecto en reposo que en imagen de redistribución mejora su perfusión en protocolos de viabilidad o con captación mayor al $50 \%$ de la zona normal del VI se considera músculo viable.

En los defectos fijos o reversibles se debe consignar su número, tamaño, intensidad y localización así como su probable relación con los territorios vasculares coronarios. En algunas ocasiones, en el estudio de estrés es posible observar dilatación transitoria del ventrículo izquierdo y acumulación pulmonar del radiofármaco. Si estos signos están presentes sin defectos de perfusión regionales se debe plantear EC multivaso balanceada; con mayor riesgo de eventos cardíacos. Estos hallazgos tienen gran especificidad incluso si la prueba es submáxima efectuada con estrés farmacológico. Se considera normal una relación de actividad miocárdica respecto del pulmón cuando es mayor de 1.8-2.0 y una dilatación transitoria menor al $20 \%$.

\section{B. Función ventricular en estudios SPECT de perfusión gatillados}

La adición simultánea de una adquisición sincronizada con el ECG del paciente, permite, sin agregar costo, tiempo de estudio ni incomodidad al paciente, evaluar la función sistólica ventricular izquierda: puede derivarse FEVI, volúmenes ventriculares y análisis de motilidad y engrosamiento parietal. Los programas automáticos disponibles están diseñados para ser utilizados con los distintos protocolos. La determinación de volúmenes ventriculares puede estar subestimada con FEVI sobre-estimada, especialmente en mujeres o niños con cavidad ventricular pequeña. Se requiere entrenamiento para un adecuado análisis del engrosamiento y de la motilidad regional, la que se altera en casos de bloqueos de rama o cirugía coronaria previa.

Otro punto en controversia en la adquisición e interpretación de los SPECT gatillados es la evaluación de función ventricular post-estrés. Esta para ser válida y representar cambios isquémicos post-estrés máximo debiera ser efectuada durante los 15-30 min posteriores, debido a que la detección de alteraciones de motilidad secundarias a atontamiento post-isquémico puede disminuir si se realiza el estudio en forma tardía. Si hay arritmias importantes, visibles en el histograma de amplitud del intervalo R-R, los parámetros funcionales son menos certeros.

C-Metabolismo con FDG: Para interpretar adecuadamente estas imágenes se requiere contar con los antecedentes clínicos y con estudios de perfusión en reposo para comparar las alteraciones regionales. Si un segmento hipoperfundido en reposo presenta normo o hipermetabolismo glucídico es considerado viable. Asimismo, un segmento hipoperfundido e hipometabólico sería no viable 


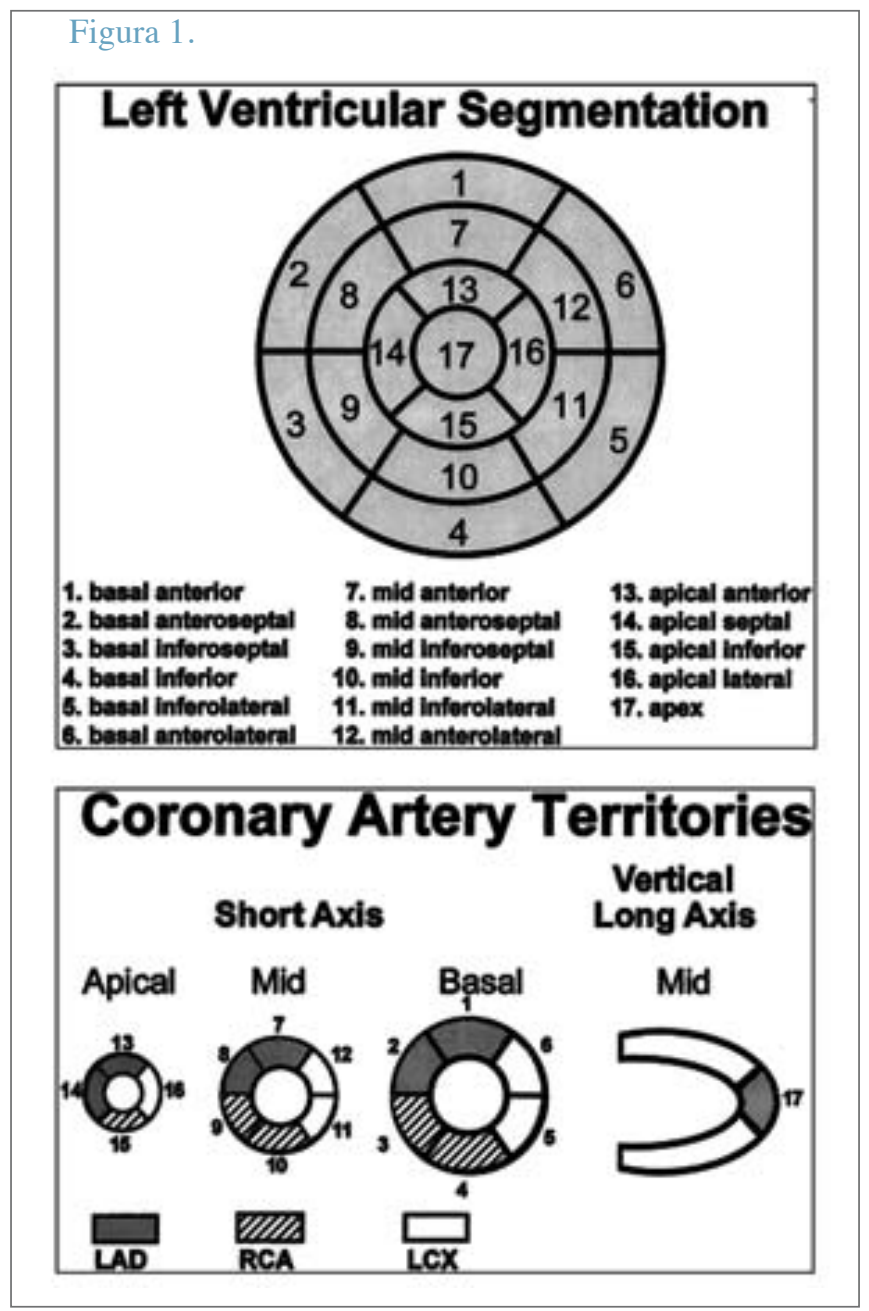

Figura 1: Asignación de territorios vasculares miocárdicos en mapa polar del ventrículo izquierdo utilizando 17 segmentos según ref. 15 modificado de ref. 24. Debe considerarse que hay variabilidad anatómica individual. (LAD: arteria descendente anterior, LCX: arteria circunfleja, RCA: arteria coronaria derecha).

(necrótico o cicatrizal). En EC crónica, esta técnica tiene mayor sensibilidad que los protocolos de viabilidad tradicionales y que la ecografía con inótropos y se considera el patrón de oro. Debe considerarse que lo que realmente confirma viabilidad funcional segmentaria es la recuperación de motilidad parietal tres meses posteriores a la revascularización.

-Los diferentes protocolos disponibles pueden responder con precisión la pregunta específica del cardiólogo: presencia, extensión y severidad de isquemia, resultado de procedimientos de revascularización, correlato anatómico (obtenido con coronariografía o angio TC) con significado funcional, clarificación de una prueba de esfuerzo no concluyente, estudio de pacientes incapaces de efectuar una prueba de esfuerzo satisfactoria o en quienes no es posible suspender terapia antianginosa, evaluación de viabilidad miocárdica y estratificación de riesgo de nuevos eventos coronarios significativos y establecer pronóstico a mediano plazo.

-Para obtener el máximo rendimiento de estos exámenes, es necesario saber interpretar adecuadamente las variantes normales, reconocer los artefactos, los errores en los procesos de adquisición y procesamiento incluyendo las limitaciones de los programas digitales de análisis disponibles, 
así como estar al tanto de las condiciones del paciente y del motivo del examen.

\section{Ventriculografía isotópica}

Se usa en algunas ocasiones para evaluar función ventricular en reposo por su gran certeza y reproducibilidad. Las indicaciones son la EC con infarto y aneurisma con indicación operatoria, las miocardiopatías isquémicas o no isquémicas, las valvulopatías, y ocasionalmente la evaluación de toxicidad por antraciclinas en pacientes oncológicos. Utiliza glóbulos rojos marcados con $\mathrm{Tc}^{99 \mathrm{~m}}$, del mismo paciente. Hay varias técnicas para esto; las más usadas son in-vivo o in-vivo in-vitro, siendo de mayor rendimiento aunque más laboriosa la in-vitro. La ventriculografía planar gatillada con la onda $\mathrm{R}$ del ECG debiera tener al menos 20 o 24 cuadros en la mejor separación septal, para calcular FEVI adecuadamente. De la vista OAI se puede calcular volúmenes ventriculares sin necesidad de muestra sanguínea. Además, es posible complementar con vistas AP y LI u OAI de $70^{\circ}$ para evaluar motilidad de las paredes anterior e inferior con mayor desproyección. La ventriculografía SPECT se procesa con programas específicos comerciales como QBS que entrega FE y volúmenes biventriculares. Existe cierta dificultad para los programas automáticos en delimitar los bordes ventriculares adecuadamente en algunos casos anormales, especialmente en ventrículo derecho. Actualmente, se considera una excelente técnica por su reproducibilidad y seguridad aunque en cierto sentido es de segunda línea; es útil cuando existen limitaciones técnicas para un ecocardiograma bidimensio- nal, la que generalmente es de elección en este contexto, por su mayor disponibilidad.

\section{Conclusiones:}

El estudio SPECT de perfusión miocárdica tiene buen rendimiento y es una técnica segura y ampliamente probada, además de cuantificable e interpretable por otro observador con una alta reproducibilidad. No tiene contraindicaciones absolutas, siendo el embarazo de tipo relativa el que debiera evitarse. La seguridad del estudio en poblaciones especiales como mujeres y ancianos también está probada. Las indicaciones, en la actualidad, están claramente establecidas ${ }^{(28-30)}$, así como la estandarización del informe o reporte ${ }^{(31,32)}$.

-El SPECT de perfusión permite evaluación funcional sin costo adicional de una adquisición sincronizada con ECG. Incluso se puede pesquisar alteración funcional ventricular izquierda sin defectos de perfusión.

-La ventriculografía isotópica también tiene un papel, especialmente por su alta (reproducibilidad

-La interpretación de los estudios debe asegurar siempre el contar con controles de calidad adecuados; ser estandarizada, entregando toda la información disponible, incluyendo las de la prueba de estrés, si la hubiera, con sus datos independientes. Además, debe tener conclusión e interpretación atingente a la solicitud clínica. Asimismo, debe aportar imágenes bien alineadas y completas para permitir reproducibilidad interobservador y comparación con eventual evaluación posterior. 


\section{Referencias:}

1. MASSARDO, T CANESSA J, SOTO JR, GONZÁLEZ P, DIGHERO H, LOBO G. Protocolos de estudios de perfusión miocárdica con radioisótopos en evaluación de cardiopatía coronaria. Rev Chil Cardiol 2004; 23: 37-44

2. HANSEN CL, MD, GOLDSTEIN RA, AKINBOBOYE OO, BERMAN DS, BOTVINICK EH, CHURCHWELL KB, et al. ASNC IMAGING GUIDELINES FOR NUCLEAR CARDIOLOGY PROCEDURES. Myocardial perfusion and function: Single photon emission computed tomography. J Nucl Cardiol 2007; 14: e39-60.

3. HENZLOVA MJ, CERQUEIRA MD, MAHMARIAN

JJ, YAO SS; Quality Assurance Committee of the American Society of Nuclear Cardiology. Stress protocols and tracers. J Nucl Cardiol. 2006; 13: e8090.

4. FOWLER M, HELLER GV. Indications for Nuclear Cardiology procedures: Suspected coronary artery disease. En: Heller GV, Hendel RC, editors. Nuclear Cardiology. Practical applications. NewYork: McGraw-Hill; 2003.1-20.

5. BAGGISH AL, BOUCHER CA. Radiopharmaceutical Agents for Myocardial Perfusion Imaging. Circulation. 2008; 118: 1668-74

6. ELHENDY AA, GREGORY SA, HOLLY TA, V. VITOLA JV. ASNC CLINICAL UPDATE Combined pharmacologic and low-level exercise stress protocols for radionuclide myocardial perfusion imaging.

Disponible en: http://www.asnc.org/imageuploads/ClinicalUpdatePharmStress2009.pdf

7. BECKMAN JA, BROWN KA, CALKINS $\mathrm{H}$, CHAIKOF E, KIRSTEN E. FLEISCHMANN KE. et al. ACC/AHA GUIDELINE ACC/AHA 2007 Guidelines on Perioperative Cardiovascular. Evaluation and Care for Noncardiac Surgery Fleisher LA, A Report of the American College of Cardiology/ American Heart Association Task Force on Practice Guidelines (Writing Committee to Revise the 2002 Guidelines on Perioperative Cardiovascular Evaluation for Noncardiac Surgery) Circulation. 2009 24; 120: e169-276.

Disponible en: http://www.asnc.org/imageuploads/ImagingGuidelinePerioperative2007.pdf

8. UNDERWOOD SR, ANAGNOSTOPOULOS C, CERQUEIRA M, ELL PJ, FLINT EJ, HARBINSON M, et al; British Cardiac Society; British Nuclear Cardiology Society; British Nuclear Medicine
Society; Royal College of Physicians of London; Royal College of Radiologists.

Myocardial perfusion scintigraphy: the evidence. Eur J Nucl Med Mol Imaging. 2004; 31: 261-91.

9. HACHAMOVITCH R, KANG X, AMANULLAH AM, ABIDOV A, HAYES SW, FRIEDMAN JD, et al. Prognostic implications of myocardial perfusion single-photon emission computed tomography in the elderly. Circulation. 2009; 120: 2197-206.

10. KANG X, BERMAN DS, LEWIN HC, COHEN I, FRIEDMAN JD, GERMANO G, et al. Incremental prognostic value of myocardial perfusion single photon emission computed tomography in patients with diabetes mellitus. Am Heart J 1999; 138: 102532.

11. HACHAMOVITCH R, BERMAN DS, SHAW LJ, KIAT H, COHEN I, CABICO JA, et al. Incremental prognostic value of myocardial perfusion single photon emission computed tomography for the prediction of cardiac death: differential stratification for risk of cardiac death and myocardial infarction. Circulation 1998; 97: 535-543.

12. POPE J.H., AUFDERHEIDE T.P. RUTHAZER R, WOOLARD RH, FELDMAN JA, BESHANSKY $\mathrm{JR}$, et al. ., Missed diagnoses of acute cardiac ischemia in the emergency department, N Engl J Med 2000; 342: 1163-1170.

13. UDELSON JE, BESHANSKY JR, BALLIN DS, FELDMAN JA, GRIFFITH JL, HANDLER J, et al., Myocardial perfusion imaging for evaluation and triage of patients with suspected acute cardiac ischemia: a randomized controlled trial, JAMA 2002; 288: 2693-2700.

14. FRUTOS E, MARÍN F, GUZMÁN M, RUIZ J, DE PABLO C, CURTO L. Utility of myocardial perfusion SPECT for evaluation of patients from Chest Pain Unit. Rev Esp Med Nucl. 2008; 27: 90-8

15. CANDELL-RIERA J, ROMERO-FARINA G, AGUADÉ-BRUIX S, CASTELL-CONESA J. La miocardiopatía isquémica desde la perspectiva de la cardiología nuclear clínica. Rev Esp Cardiol. 2009; 62: 903-17

16. GERMANO G, KIAT H, KAVANAGH PB, MORIEL M, MAZZANTI M, SU HT, et al. Automatic quantification of ejection fraction from gated myocardial perfusion SPECT. J Nucl Med. 1995; 36: 2138-47.

17. FABER TL, COOKE CD, FOLKS RD, VANSANT JP, NICHOLS KJ, DEPUEY EG, et al. Left ventricular function and perfusion from gated SPECT perfusion images: an integrated method. J Nucl Med. 
1999; 40: 650-9.

18. CERQUEIRA MD, WEISSMAN NJ, DILSIZIAN V, JACOBS AK, KAUL S, LASKEY WK, et al. Standardized myocardial segmentation and nomenclature for tomographic imaging of the heart: a statement for healthcare professionals from the Cardiac Imaging Committee of the Council on Clinical Cardiology of the American Heart Association. Circulation 2002 ;105: 539-42.

19. SHARIR T, GERMANO G, KANG X, LEWIN HC, MIRANDA R, COHEN I, et al. Prediction of myocardial infarction versus cardiac death by gated myocardial perfusion SPECT: risk stratification by the amount of stress-induced ischemia and the poststress ejection fraction. J Nucl Med. 2001; 42: 831-7

20. ABIDOV A, SLOMKA PJ, NISHINA H, HAYES SW, KANG X, YODA S, et al. Left ventricular shape index assessed by gated stress myocardial perfusion SPECT: initial description of a new variable.J Nucl Cardiol. 2006; 13: 652-9.

21.GERMANO G, KAVANAGH PB, SLOMKA PJ, VAN KRIEKINGE SD,POLLARD G, BERMAN DS. Quantitation in gated perfusion SPECT imaging: The Cedars-Sinai approach. J Nucl Cardiol 2007; 14: 433-54

22. LETTE J, TATUM JL, FRASER S, MILLER DD, WATERS DD, HELLER G, et al. Safety of dipyridamole testing in 73,806 patients: the Multicenter Dipyridamole Safety Study. J Nucl Cardiol 1995; 2: 3-17

23. MEYERS AM, TOPHAM L, BALLOW J, TOTAH D, WILKE R. Adverse Reactions to Dipyridamole in Patients Undergoing Stress/Rest Cardiac Perfusion Testing J Nucl Med Technol 2002; 30: 21-4

24. EINSTEIN AJ, MOSER KW, THOMPSON RC, CERQUEIRA MD, HENZLOVA MJ. Radiation dose to patients from cardiac diagnostic imaging. Circulation 2007; 116: 1290-305.

25. CHANG SM, NABI F, XU J, RAZA U, MAHMARIAN JJ. Normal stress-only versus standard stress/ rest myocardial perfusion imaging similar patient mortality with reduced radiation exposure. J Am Coll Cardiol 2010; 55: 221-30

26. EVANGELISTA L, ACAMPA W, PETRETTA M, FERRO A, RICCI F, LUONGO L, et al. Incremental prognostic value of cardiac single-photon emission computed tomography after nitrate administration in patients with ischemic left ventricular dysfunction. $\mathrm{J}$ Nucl Cardiol 2009; 16: 38-44.

27. LUCIGNANI G, CUOCOLO A. Recent advances in the assessment of myocardial viability. Eur J Nucl Med Mol Imaging 2009; 36: 1892-95

28. HUSAIN SS. Myocardial Perfusion Imaging Protocols: Is There an Ideal Protocol? J Nucl Med Technol 2007; 35: 3-9. Disponible en: http://tech.snmjournals.org/cgi/content/full/35/1/3

29. Imaging guidelines for nuclear cardiology procedures, part 2. American Society of Nuclear Cardiology. J Nucl Cardiol. 1999; 6: G47-G84.

30. HENDEL RC, BERMAN DS, DI CARLI MF, HEIDENREICH PA, HENKIN RE, PELLIKKA PA, et al. ACCF/ASNC/ACR/AHA/ASE/SCCT/SCMR/ SNM 2009 appropriate use criteria for cardiac radionuclide imaging: A report of the Am Coll Cardiol Foundation Appropriate Use Criteria Task Force. Circulation 2009; 119: e561-87.

31. TILKEMEIER PL, COOKE CD, FICARO EP, GLOVER DK, HANSEN CL, MCCALLISTER BD JR. American Society of Nuclear Cardiology information statement: Standardized reporting matrix for radionuclide myocardial perfusion imaging. J Nucl Cardiol. 2006; 13: e157-71.

32. TILKEMEIER PL, COOKE CD, GROSSMAN GB, MCCALLISTER BD JR, WARD RP. Standardized Reporting of Radionuclide Myocardial Perfusion and Function. J Nucl Cardiol 2009:16: 650. Disponible en: http://www.asnc.org/imageuploads/Imaging Guidelines Reporting July 2009.pdf (doi:10.1007/ s12350-009-9095-8)

33. BOTVINICK E. Current Methods of Pharmacologic Stress Testing and the Potential Advantages of New Agents. J Nucl Med Technol 2009; 37: 14-25 


\section{Anexo:}

\section{SPECT DE PERFUSIÓN MIOCÁRDICA}

- Ayuno mínimo de $4 \mathrm{~h}$. En diabéticos insulino dependientes, debe compatibilizarse con esquema de tratamiento.

- No ingerir xantinas, café, té, chocolate, mate, ni bebidas que contengan cola en las $24 \mathrm{~h}$ previas al estudio (con protocolo de vasodilatadores).

- La eventual suspensión de medicamentos que puedan interferir con los resultados de la prueba, debiera consultarse con el médico tratante.

- Todo procedimiento debe ser permanentemente supervisado por personal con entrenamiento en resucitación cardiopulmonar y tener disponibles los insumos y equipos necesarios para la atención de eventuales emergencias.

- Durante el estrés físico o farmacológico debe controlarse la frecuencia cardiaca y la presión arterial con registro ECG continuo.

\section{AGENTES VASODILATADORES}

El dipiridamol inhibe la recaptación celular de la adenosina de las células miocárdicas, produciendo un incremento en sus niveles plasmáticos, con un importante efecto vasodilatador no selectivo. El dolor precordial no necesariamente representa isquemia y puede ser explicado por robo subendocárdico. Hay nuevos vasodilatadores específicos, con menores efectos colaterales no usados aún rutinariamente en nuestro medio (33).

Contraindicaciones de los vasodilatadores

- Broncoespasmo

- Asma severa o asociada con hipertensión pulmonar

- Hipotensión

- Hipersensibilidad al dipiridamol o a la adenosina

- Pacientes que requieran medicación continua con xantinas

- Bloqueo AV de $2^{\circ}$ o $3^{\circ}$ grado (sin marcapasos)

- Infarto de miocardio reciente, complicado

- Angina inestable

- Estenosis aórtica severa

- Cardiomiopatía hipertrófica obstructiva

- Hipotensión ortostática severa

\section{Efectos secundarios:}

- Dolor torácico o angina

- Mareo
- Cefalea

- Anormalidades ECG (bloqueo AV exclusivamente para el caso de la adenosina-)

- Enrojecimiento facial

Náuseas y vómito

Dolor abdominal

Broncoespasmo

Hipotensión severa

Malestar inespecífico

\section{Protocolo de infusión de dipiridamol}

- Administrar 0,56 mg/kg por vía iv, durante $4 \mathrm{~min}$. Se puede utilizar una bomba de infusión o un goteo continuo o una inyección lenta con la droga diluida, (ej. $8 \mathrm{ml}$ de suero fisiológico, a razón de $2 \mathrm{ml} / \mathrm{min}$ ).

- Administrar el trazador 2 a 4 min después de finalizada la infusión de dipiridamol. Puede hacerse una segunda infusión de $2 \mathrm{~min}$, iniciando 2 min después de finalizada la primera, empleando la mitad de la dosis o usar $0.84 \mathrm{mg} / \mathrm{kg}$ durante $4 \mathrm{~min}$.

- Inyectar 50-100 mg de aminofilina IV en $30 \mathrm{seg}$ en pacientes que desarrollen síntomas significativos después de la administración del radiofármaco. Cuando los síntomas ocurran antes de administrarlo, éste deberá inyectarse inmediatamente, esperando 1 min antes de inyectar la aminofilina. Puede aumentarse esta dosis.

- Algunos centros usan aminofilina de manera rutinaria. La vida media plasmática de la aminofilina es más corta que la del dipiridamol, por lo que se puede requerir dosis adicionales.

\section{AGENTES INOTRÓPICOS}

El más empleado es la dobutamina, amina simpaticomimética, sintética que incrementa el flujo coronario aproximadamente al doble del nivel de reposo. (vida media biológica en plasma de dos minutos). Su empleo se reserva para casos que requieran estrés farmacológico y no se puedan emplear vasodilatadores.

Contraindicaciones para el uso de inotrópicos

- Taquicardia ventricular

Bloqueos $\mathrm{AV}$ de $2^{\circ}$ o $3^{\circ}$ grado

- Fibrilación auricular con respuesta ventricular rápida

- Extrasístoles ventriculares frecuentes o complejas

- Angina inestable

- Cardiomiopatía obstructiva o hipertrófica

- Infarto agudo de miocardio 
- Hipertensión arterial severa Efectos secundarios:

- Dolor torácico

- Disnea o palpitaciones

- Náuseas y vómito

- Cefalea

- Arritmias ventriculares o supraventriculares

- Hipo o hipertensión

- Cambios electrocardiográficos

Protocolo de Infusión de Dobutamina

- Suspender betabloqueadores durante $48 \mathrm{~h}$

- Administrar la droga iniciando goteo a $5 \mu \mathrm{g} / \mathrm{kg} / \mathrm{min}$ por bomba de infusión y aumentando a 10,20,30 y $40 \mu \mathrm{g} / \mathrm{kg} / \mathrm{min}$ cada tres min o según preferencia del cardiólogo a cargo de acuerdo al paciente

- Cuando no se alcance un adecuado incremento de la frecuencia cardiaca, se debe administrar $1 \mathrm{mg}$ de atropina por vía SC, manteniendo la infusión de dobutamina a $40 \mu \mathrm{g} / \mathrm{kg} / \mathrm{min}$ por $3 \mathrm{~min}$ adicionales.

- En pacientes inestables se recomienda que los incrementos se realicen a intervalos de $5 \mu \mathrm{g} / \mathrm{kg} / \mathrm{min}$ cada tres min.

- Administrar el radiotrazador cuando se alcance la frecuencia cardiaca máxima, continuando la infusión de dobutamina por $2 \mathrm{~min}$.

- Si se presentan efectos secundarios puede revertirse la acción farmacológica de la droga con betabloqueadores de acción rápida. Controlar por $5 \mathrm{~min}$ adicionales al término de la infusión. 